\title{
Treatment of Severe, Treatment- Refractory Obsessive-Compulsive Disorder: A Study of Inpatient and Community Treatment
}

\author{
By Mark J. Boschen, PhD, MAPS, Lynne M. Drummond, MRCP, FRCPsych, \\ and Anusha Pillay, BA (Hons), MSc
}

\begin{abstract}
Introduction: This research reports on a prospective outcome study of two cohorts of patients with severe, chronic, resistant obsessive-compulsive disorder (OCD).

Methods: One cohort consisted of a total of 52 patients treated in an inpatient setting, while the second group comprised 65 patients treated in a community-outpatient setting. Treatment consistent primarily of intensive graded exposure and self-imposed response prevention augmented with cognitive restructuring.
\end{abstract}

Results: The groups demonstrated significant improvement over the course of treatment. In the inpatient and community groups, there was significant improvement over the first 12 weeks of treatment, and further improvement between 12 and 24 weeks.

Conclusion: These results suggest that even

\section{FOCUS POINTS}

- Obsessive-compulsive disorder (OCD) is a severe, chronic complaint that significant impacts on functioning and quality of life.

- Despite the existence of effective treatments for OCD, there are many for whom treatment is ineffective.

- Some individuals present with OCD that is resistant to treatment with pharmacologic and behavioral therapies.

- Even for the most severe, treatment-resistant cases, inpatient and community treatment with a specialist treatment team can be effective.

for patients who have demonstrated treatmentresistance, there may be benefit in intensive behavioral treatment of OCD. In addition it was found that even for those patients with the most profound refractory OCD and complicating factors inpatient stays of up to 24 weeks were effective in reducing symptoms.

CNS Spectr. 2008;13(12):xxx-xx

\footnotetext{
Dr. Boschen is lecturer in clinical psychology in the School of Psychology at Griffith University in Southport, Australia. Dr. Drummond is consultant psychiatrist and senior lecturer at St George's, University of London. Ms. Pilay is senior cognitive behavioural psychotherapist at South West London and St George's Mental Health National Health Service Trust in the United Kingdom.

Faculty Disclosures: The authors do not have an affiliation with or financial interest in any organization that might pose a conflict of interest.

Submitted for publication: xxx, 2008; Accepted for publication: xxx, 2008.

Please direct all correspondence to: Mark J. Boschen, PhD, MAPS, School of Psychology, Griffith University, Parklands Drive, Southport 4215, Australia; E-mail: M.Boschen@griffith.edu.au.
} 


\section{INTRODUCTION}

Obsessive-compulsive disorder (OCD) is a condition characterized by recurrent intrusive ideas, impulses, or urges (obsessions), along with overt or covert behaviors (compulsions) aimed at reducing the distress these obsessions cause. ${ }^{1}$ Lifetime prevalence rates for OCD are estimated at $1.6 \%$, ${ }^{2}$ with 1-year prevalence estimated at $0.8 \%,{ }^{3}$ and point-prevalence at $\sim 0.4 \% .{ }^{4}$ OCD causes significant distress and impairment in social $\left.\right|^{5}$ and occupational ${ }^{6}$ functioning, as well as reduced quality of life..$^{7-9}$ Research into OCD has continued to expand over the last 3 decades, attracting more research than any anxiety disorder other than posttraumatic stress disorder. ${ }^{10}$ Treatment of OCD is efficacious. In a meta-analysis, Eddy and colleagues $^{11}$ found that both pharmacotherapy with serotonin reuptake inhibitors (SRIs) and cognitivebehavioral interventions yielded large effect sizes, ranging from 1.18 for pharmacotherapy to 1.72 for combined cognitive-behavioral therapy (CBT) and pharmacotherapy. Despite these results, it is known that $\sim 38 \%$ of patients are treatment refractory, not experiencing clinically significant benefit $^{12}$ from treatment, and $<30 \%$ of patients were asymptomatic following treatment. ${ }^{13}$

There is a growing realization of the need to report on the effectiveness of interventions delivered in routine clinical practice, in addition to well-controlled efficacy studies, ${ }^{14-16}$ for example, in individuals with comorbid conditions commonly seen in epidemiological studies. ${ }^{17}$ Furthermore, there is increasing acceptance of the need to assess outcome using several different methods beyond simple significance tests of pre-post symptom severity. Assessment of each individual for reliable and clinically significant change ${ }^{12,18}$ allows the investigator to gauge information beyond analysis of group means, potentially revealing rich information about the responses of individual patients.

There are few reports of CBT outcome studies in cases of severe OCD, with our literature search finding only two similar investigations. One previous report on the outcome of behavioral treatment of extremely severe, treatment-resistant OCD was published by Drummond. ${ }^{19}$ In this cohort of 53 inpatients, the author reported a $40 \%$ reduction in OCD symptoms occurring in $>60 \%$ of those treated. Furthermore, the gains from inpatient treatment were maintained when the cohort was examined at an 18-month follow-up. An inpatient behavioral-therapy unit in which most of the patients were diagnosed with OCD was also described by Thornicroft and colleagues. ${ }^{20}$ Though these patients were reported as experiencing high levels of discomfort and handicap from their OCD symptoms, as well as significant improvement from treatment, they were not assessed with a psychometrically validated rating scale, making comparisons with the current cohort difficult. Stuart and colleagues ${ }^{21}$ also reported on intensive residential treatment of severe OCD, although their patients had considerably lower severity that those treated by Drummond and colleagues ${ }^{19}$ or patients in the current study.

The current prospective study aims to assess the effectiveness of treatment for severe OCD in clinical practice, using cognitive, behavioral and pharmacologic treatments. It examines the outcome of treatment in both inpatient and community settings, using an assortment of complementary outcome criteria. The outcomes are then benchmarked against the treatment effects reported in previous efficacy studies. It expands on previous research in three important ways. It is the first study to examine outcome treatment, predominantly using $\mathrm{CBT}$, in a cohort of severe, treatment-refractory patients seen in both inpatient and community settings. Second, it reports on routine clinical practice data allowing for examination of the effectiveness (as opposed to the efficacy) of treatment for OCD. Third, it examines the individual outcomes of patients in both treatment settings.

\section{STUDY ONE: INPATIENT TREATMENT-METHODS}

\section{Participants}

A total of 52 patients were admitted to the residential unit of the National Service for the Treatment of Severe, Chronic Refractory ObsessiveCompulsive and Body Dysmorphic Disorders in the Behavioural and Cognitive Psychotherapy Unit (BCPU) of South West London and St George's Mental Health Trust between April 2006 and December 2007 for treatment of OCD. These were consecutive patients to be admitted to the Unit, who were suffering from OCD as their primary diagnosis. This treatment unit acts as a tertiary (and higher) referral service for patients who have not responded to treatment elsewhere. Patients admitted to the BCPU are required to have a YaleBrown Obsessive Compulsive Scale (Y-BOCS) ${ }^{22,23}$ score of $\geq 30$ which represents profoundly severe 
OCD. In addition they are expected to have undergone psychopharmacologic treatment with at least two SRIs. Furthermore, they were required to have been offered augmentation of their SRIs with either supranormal dosages, by the addition of a dopamine antagonist, or by the use of mood stabilizers (as recommended by Pallanti and colleagues ${ }^{24}$ ), with unsatisfactory response or to have been offered this treatment and been unable to tolerate. Each patient is also required to have demonstrated poor response to two trials of CBT in an outpatient setting (generally including one home-based outpatient intensive treatment). Patients who meet these basic severity markers are suitable for treatment in part of the National Consortium of OCD services which has been described elsewhere. ${ }^{25,26}$ To be suitable for inpatient treatment, there needs to be a reason why 24-hour nursing care is necessary. Reasons for the requirement of inpatient treatment include being a danger to themselves (through suicide risk, or self-neglect), being a danger to others (eg, through risk of impulsive acts), taking at least 3 hours in performing compulsions while getting out of bed, delayed sleep-phase shift, or complicating comorbid diagnoses.

\section{Assessment of Psychopathology}

All patients were assessed by a qualified psychiatrist with $>20$ years of experience in OCD prior to entry to the unit to confirm their diagnoses. Outcome was assessed using a set of clinician rated and self-report measures. The primary treatment outcome measure used for all patients was the Y-BOCS, ${ }^{22,23}$ a clinician-rated measure of severity of obsessive-compulsive symptoms, based on a semi-structured clinical interview with the patient. The Y-BOCS is recognized as the gold standard in outcome assessment for OCD, with strong psychometric properties, and is also well suited for use in evaluating individual clinically significant change. ${ }^{13}$ The Padua Inventory $(\mathrm{PI})^{27}$ was used as a self-report measure of obsessions and compulsions. It has sound reliability, and validity for use in clinical populations. The Beck Depression Inventory $(B D I)^{28}$ was used as a self-report measure of severity of depressive symptoms. Since its original publication, ${ }^{29}$ the BDI has seen widespread use as an outcome measure in inpatient and outpatient studies, and has strong psychometric properties. ${ }^{30}$

\section{Procedure}

Treatment is provided at the BCPU inpatient unit by a multidisciplinary team consisting of psy- chiatrists, cognitive-behavioral psychotherapists, occupational therapists, and psychiatric nurses. Patients are admitted for a maximum of 6 months, during which they receive intensive treatment consisting of both psychopharmacologic and cognitive-behavioral interventions. A strong emphasis is placed on the use of exposure and response prevention (ERP) methods. Each patient is allocated a therapist who collaboratively works with the patient to decide the treatment program. This programme is then overseen by the ward-based nursing staff on a daily basis. Due to the severity of the condition of patients, individual and group occupational-therapy sessions focus on activities of daily living, as well as anxiety management and exposure-therapy groups.

\section{RESULTS}

A total of 52 patients were admitted over the study period. Demographic characteristics of the sample are described in Table 1. Following admission, patients had a medication review. In general, the emphasis was on trying to keep their medication regime as simple as possible, and aimed at prescribing the lowest possible dose compatible with health and symptom reduction. Patients were admitted for a mean of $135.51 \pm 59.62$ days. Missing data was handled by carrying the last observation forward. In cases where there was no earlier data to carry forward, subsequent missing datapoints were allowed to remain missing in the analyses.

\section{Symptom Change During Treatment}

The primary treatment outcome measure used was the Y-BOCS. For patients with missing data (including those who dropped out of treatment), the last observation was carried forward for use in the analysis of the statistical significance of the intervention. Analysis of variance revealed significant differences between Y-BOCS scores at admission, 12 weeks, and 24 weeks $[F(2,90)=46.62$, $P<.001]$. Follow-up comparisons between consecutive measurements demonstrated significant falls in total Y-BOCS scores from admission to 12 weeks $\left(\mathrm{M}_{\text {Pre }}\right.$ : 35.30, $\mathrm{M}_{12 \mathrm{Wks}}: 30.28,95 \% \mathrm{Cl}_{\text {Diff: }}$ 2.96 to $7.08, t=4.91, \mathrm{df}=45, P<.001)$ and between the 12-week and 24-week assessments $\left(\mathrm{M}_{24 \mathrm{Wks}}\right.$ : 24.37, 95\% Cl $\mathrm{l}_{\text {Diff: }} 3.86$ to 7.97, $t=5.80, d f=45, P<.001$ [Figure 1]). Overall, this represented a drop of $31 \%$ in Y-BOCS scores for the total group from admission to 24 weeks. A comparison between the length of inpatient admission and the drop in 
Y-BOCS scores is provided in Figure 2.

The PI was used as a self-report measure of treatment outcome, with results supporting those gained from the clinician-ratedY-BOCS. Scores on the PI were significantly different across the three assessment timepoints $[F(2,82)=16.83, P<.001]$, with significant drops between admission and 12-week assessment ( $\mathrm{M}_{\text {Pre }}$ : 88.66, $\mathrm{M}_{12 \mathrm{Wks}}$ : 76.45, $95 \% \mathrm{Cl}_{\text {Diff: }} 2.89$ to $\left.21.54, t=2.65, d f=41, P<.05\right)$ and again between 12- and 24-week assessments $\left(\mathrm{M}_{24 \mathrm{Wks}}: 60.69,95 \% \mathrm{Cl}_{\text {Diff: }} 8.84\right.$ to $22.69, t=4.60$, $d f=41, P<.001)$. Overall, this represented a drop of $32 \%$ in PI scores from admission to 24-week assessment.

Treatment was also associated with a significant reduction in depression symptoms, with an overall significant difference between BDI scores at admission, 12 weeks and 24 weeks [ $F(2$, 86) $=15.59, P<.001]$. Post-hoc testing revealed that there were significant drops in total BDI scores between admission and 12-week assessment ( $\mathrm{M}_{\text {Pre: }}$ :27.89, $\mathrm{M}_{12 \mathrm{Wks}}$ : 24.18, 95\% $\mathrm{Cl}_{\text {Diff: }} 0.78$ to 6.63 , $t=2.55, d f=43, P<.05)$, and between 12 week and 24 week assessment $\left(\mathrm{M}_{24 \mathrm{Wks}}: 19.45,95 \% \mathrm{Cl}_{\text {Diff }}\right.$ :
1.93 to $7.52, t=3.41, d f=43, P<.005)$. The fall in the Y-BOCS and BDI scores in the inpatient group is shown in Figure 1, while the decline in PI scores is depicted in Figure 3. Overall, this represented a drop of $30 \%$ in BDI scores from admission to 24week assessment.

\section{Evaluation of Individuals' Reliable and Clinically Significant Change}

In addition to the evaluation of overall change in group mean symptoms, each individual's scores on the Y-BOCS were assessed according to the criteria of Jacobson and Truax ${ }^{12}$ for reliable and clinically significant change. We followed the approach of Fisher and Wells ${ }^{13}$ who suggested that a change $\geq 10$ points on the $Y$-BOCS constituted a reliable change according to the Jacobsen and Truax ${ }^{12,18}$ criteria. To demonstrate clinically significant change according to these criteria, individuals are also required to end treatment with symptom severity closer to the normal than the clinical mean. Again, we adopted the cutoff used by Fisher and Wells, ${ }^{13}$ which stipulates that a score $\leq 14$ on the $Y$-BOCS reflects an individual

\begin{tabular}{|c|c|c|}
\hline & Inpatient (Study 1; $N=52$ ) & Community (Study 2; $N=62$ ) \\
\hline \multirow[t]{2}{*}{ Sex } & $29(56 \%)$ male & $29(47 \%)$ male \\
\hline & $23(44 \%)$ female & $33(53 \%)$ female \\
\hline Age at admission (years), mean & $35.17 \pm 11.52$ & $39.12 \pm 11.83$ \\
\hline Age of onset of OCD (years), mean & $19.43 \pm 11.24$ & $20.52 \pm 8.61$ \\
\hline Duration of OCD (years), mean & $16.76 \pm 9.76$ & $18.66 \pm 11.18$ \\
\hline \multirow[t]{4}{*}{ Marital status } & $38(73 \%)$ unmarried & $52(84 \%)$ unmarried \\
\hline & $10(19 \%)$ married/cohabiting & $6(10 \%)$ married/cohabiting \\
\hline & $3(6 \%)$ divorced/separated & $4(7 \%)$ divorced/separated \\
\hline & 1 unrecorded & \\
\hline \multirow[t]{5}{*}{ Employment status } & 28 (82) unemployed & $32(52 \%)$ unemployed \\
\hline & $1(3 \%)$ full-time work & $30(48 \%)$ full-time Work \\
\hline & $3(9 \%)$ part-time/volunteer work & \\
\hline & $2(6 \%)$ students & \\
\hline & 18 unrecorded & \\
\hline Duration of stay, mean & $136 \pm 59$ days & \\
\hline \multirow[t]{3}{*}{ Y-BOCS Total Score, mean } & $M_{\text {Pre }}: 35.30 \pm 4.18$ & $\mathrm{M}_{\text {Pre }}: 27.81 \pm 5.87$ \\
\hline & $\mathrm{M}_{12 \mathrm{Wks}}: 30.28 \pm 8.11$ & $\mathrm{M}_{12 \mathrm{Wk}}: 21.78 \pm 7.28$ \\
\hline & $\mathrm{M}_{24 \mathrm{Wks}}: 24.37 \pm 10.62$ & $\mathrm{M}_{24 W \mathrm{ks}}: 19.27 \pm 7.96$ \\
\hline \multicolumn{3}{|c|}{$O C D=0 b s e s s i v e-$ compulsive disorder; $Y-B O C S=Y a l e-B r o w n$ Obsessive-Compulsive Scale. } \\
\hline \multicolumn{3}{|c|}{ Boschen MJ, Drummond LM, Pillay A. CNS Spectr. Vol 13, No 12. 2008.} \\
\hline
\end{tabular}


being closer to the normal than the clinical population. Fisher and Wells also suggested that a score $\leq 7$ on the $\mathrm{Y}$-BOCS is indicative of recovery from OCD.

In our inpatient sample, 46 patients hadY-BOCS scores which were used to evaluate change. Of these, 20 (44\%) experienced statistically reliable improvement, and 10 (22\%) also experienced a fall to below the cutoff score for clinically significant improvement. No patients demonstrated reliable deterioration. As would be expected in a severe, treatment-refractory sample, the number of individuals experiencing recovery from OCD symptoms was small, with only 2 (4\%) dropping to $\leq 7$ on the Y-BOCS.

An alternative criterion of a drop $\geq 25 \%$ on the Y-BOCS has also been specified as a definition of clinically significant treatment response by Pallanti and colleagues. ${ }^{24}$ According to this alternative criteria, 27 (52\%) of our cohort demonstrated a clinically significant treatment response.

\section{Benchmarking the Inpatient Treatment Program}

Previous authors have compared the results of treatments conducted in carefully controlled research studies with those undertaken in routine clinical practice. Such comparisons allow the researcher to examine the effectiveness and as

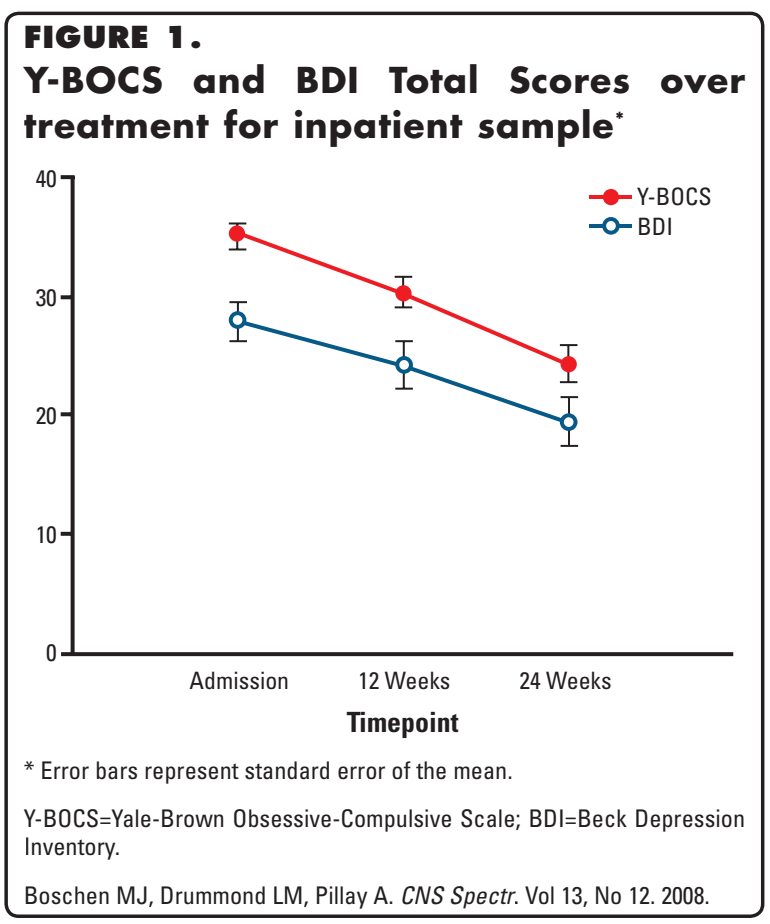

the efficacy of the intervention. The uncontrolled effect size of the inpatient treatment was 1.35. This is compared to the pre-post treatment effect sizes reported in the meta-analysis of OCD treatments by Eddy and colleagues ${ }^{11}$ in Figure 4. The number of inpatients who experienced significant improvement in their symptoms, and those who experienced recovery are compared with the results of Fisher and Wells ${ }^{13}$ in Figure 5.

\section{STUDY TWO: COMMUNITY TREATMENT-METHODS}

\section{Participants}

The Trustwide tertiary referral service of South West London and St George's Mental Health NHS Trust accepted 62 patients for community-based treatment of severe, OCD between April 2006 and December 2007. All patients were required to have experienced unsatisfactory therapeutic response to CBT provided by their local mental health team. In addition, it was expected that the patients had been offered SRI medication. Furthermore, many had already been offered psychopharmacologic augmentation of this regimin according to the recommendations of Pallanti and colleagues. ${ }^{24}$ All patients were judged by the treating team to have demonstrated no clinically significant improvement from previous treatment regimes. The criteria employed for entry to the service are therefore

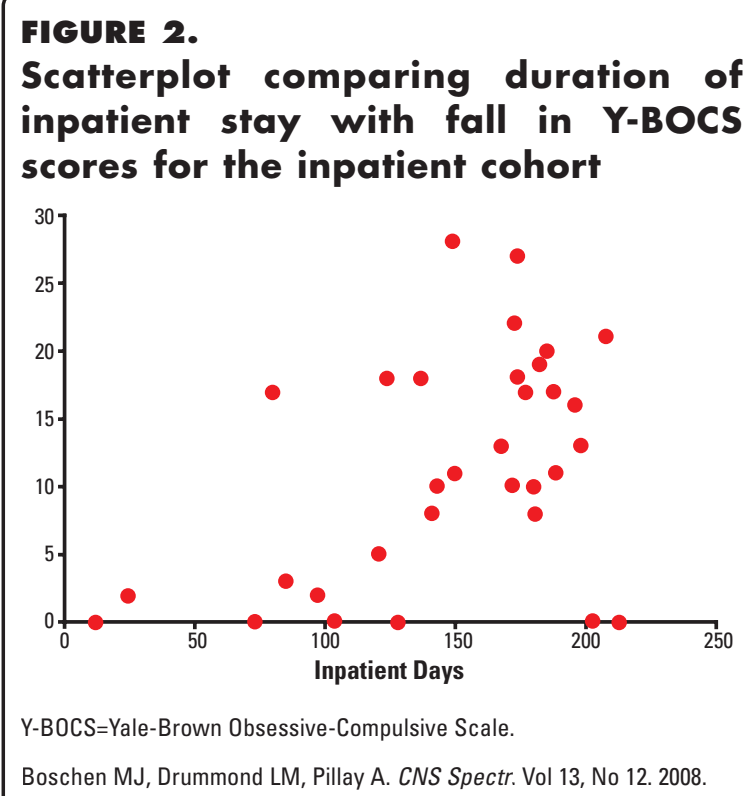


not as stringent as for those admitted to the inpatient unit, but individuals are still expected to demonstrate a considerable resistance to improvement from previous treatment. Upon referral, cases were discussed with the treating team and interventions planned. The details of the operation of this service are described elsewhere. ${ }^{31}$ Treatment is provided to individuals from the five London boroughs of Kingston, Merton, Richmond, Sutton, and Wandsworth. These boroughs have a total adult population of $\sim 1$ million. Treatment consists of combined behavioral, cognitive, and psychopharmacologic interventions, with a strong emphasis on the use of ERP. Home-based treatment involving ERP is usually offered during therapy. The community treatment differs from other outpatient facilities in that it is staffed by therapists with expertise in CBT for OCD. It also aims to provide input into cases meeting Level 5 of the NICE guidelines for treatment of OCD, ${ }^{32}$ while also advising other treatment services catering for Levels 1-4.

\section{RESULTS}

Demographic details of the 62 successive referrals used in the community sample are reported in Table 1. Change in medication regime occurred in a very small number of patients, with one patient receiving a new prescription for an SRI, and two having a dopamine antagonist added to their existing SRI. Missing data

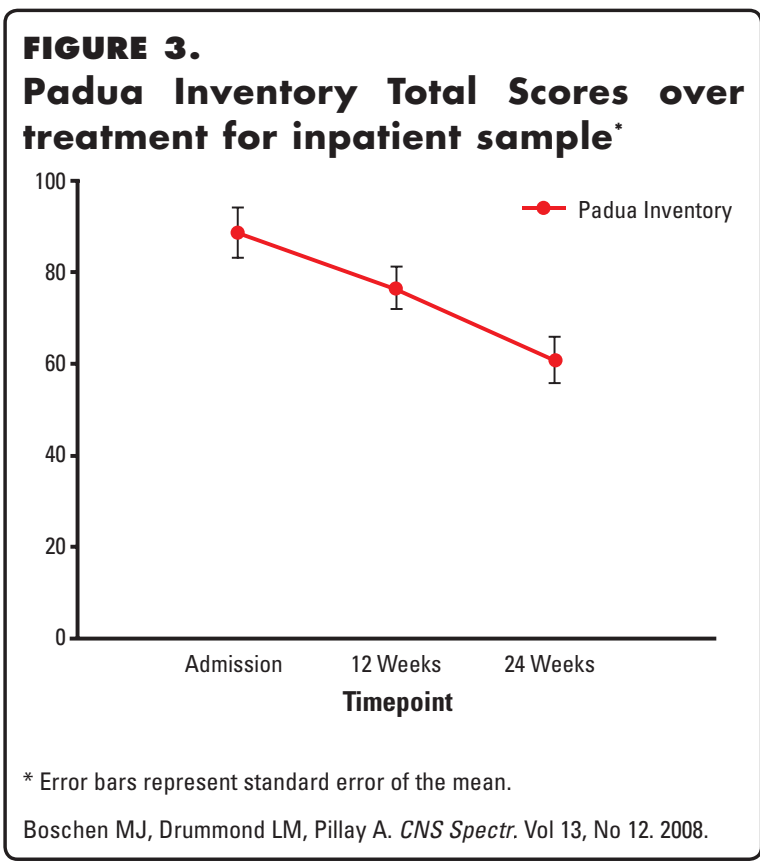

was handled where possible by carrying the last observation forward to subsequent measurements. Where no earlier data was available this data remained missing for subsequent statistical analyses.

\section{Symptom Change During Treatment}

Total scores on the Y-BOCS were significantly different between the three assessment timepoints of pre-treatment, 12 weeks, and 24 weeks $[F(2,122)=65.32, P<.001]$. The community group's Y-BOCS scores declined significantly between pre-treatment and 12-week assessment $\left(\mathrm{M}_{\text {Pre }}\right.$ : 27.81, $\mathrm{M}_{12 \mathrm{Wks}}: 21.78,95 \% \mathrm{Cl}_{\text {Diff }}: 4.63$ to 7.42 , $t=8.63, d f=61, P<.001)$, as well as between 12 and 24-week assessment $\left(\mathrm{M}_{24 \mathrm{Wks}}: 19.27,95 \% \mathrm{Cl}_{\text {Diff: }}\right.$ : 1.26 to $3.76, t=4.01, d f=61, P<.001)$. This represents a reduction of $21 \%$ in clinician-rated OCD symptoms.

When results of the self-report PI were examined, there was an overall significant difference between the timepoints $[F(2,114)=16.50, P<.001]$, and differences between the baseline and 12week assessment ( $\mathrm{M}_{\text {Pre }}$ : 77.07, $\mathrm{M}_{12 \mathrm{Wks}}: 67.66,95 \%$ $\mathrm{Cl}_{\text {Diff: }} 3.58$ to $\left.15.24, t=3.23, d f=57, P<.005\right)$, and the 12 and 24-week assessments $\left(\mathrm{M}_{24 \mathrm{Wks}}\right.$ : 59.97, 95\% $\mathrm{Cl}_{\text {Diff: }} 1.78$ to $\left.12.12, t=2.69, d f:=58, P<.01\right)$. This represents a drop of $12 \%$ in self-reported OCD symptoms.

Depression symptoms, as assessed by the BDI were different across the three timepoints $[F(2$, $120)=30.66, P<.001]$, with significant reductions between the baseline and 12-week assessments $\left(\mathrm{M}_{\text {Pre }}: 24.92, \mathrm{M}_{12 \mathrm{Wks}}: 19.25,95 \% \mathrm{Cl}_{\text {Diff: }} 3.56\right.$ to 7.79 ,

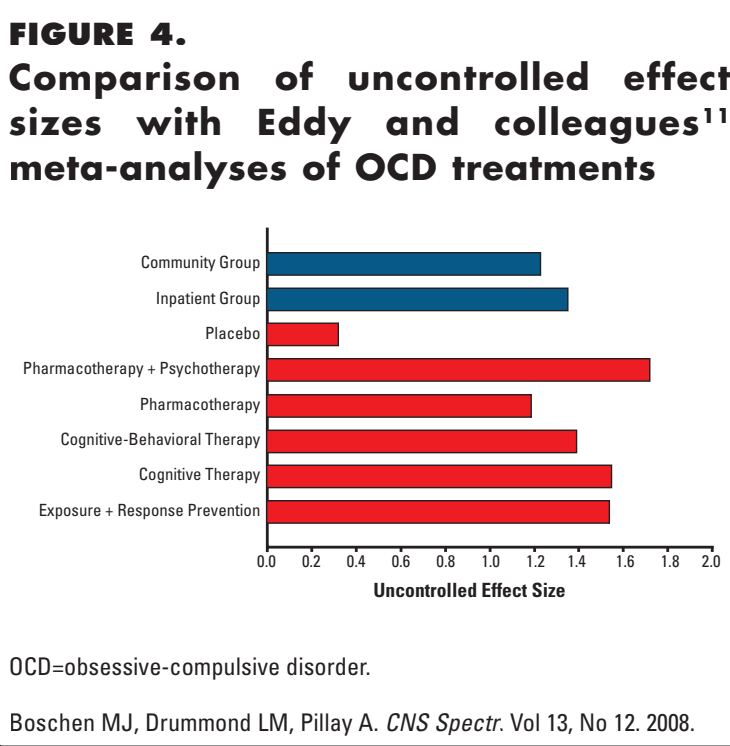


$t=5.37, d f=60, P<.001)$, and a further fall between 12 and 24 weeks $\left(\mathrm{M}_{24 \mathrm{Wks}}: 17.26,95 \% \mathrm{Cl}_{\text {Diff }}: 0.46\right.$ to $3.57, t=2.59, d f=61, P<.05)$. This represents a reduction of $23 \%$ in self-reported depression symptoms. Mean scores on the three outcome measures are shown in Figures 6 and 7.

\section{Evaluation of Individuals' Reliable and Clinically Significant Change}

Using the same criteria as in Study One, YBOCS total scores for each community patient were examined individually to assess for reliable and clinically significant change, as well as whether decline in symptoms left the patient asymptomatic following treatment. In the community sample, 62 patients had initial Y-BOCS scores that allowed for assessment of reliable and clinically significant change. Of these, 25 $(40 \%)$ experienced a decline $\geq 10$ points on the Y-BOCS, while 12 (19\%) met both the required criteria of a fall of 10 points and a final score $\leq 14$ on the Y-BOCS. No patients reliably deteriorated. Only three patients (5\%) were asymptomatic ("recovered"), with Y-BOCS scores $\leq 7$, by the end of treatment. According to the alternative Pallantiand colleagues ${ }^{24}$ criterion for significant clinical change, 24 (39\%) of patients demonstrated a significant treatment response.

\section{Benchmarking the Community Treatment Program}

In an effort to compare the results gained from the outpatient treatment program, the uncontrolled pre-post effect size measurement from the Y-BOCS was compared with results reported

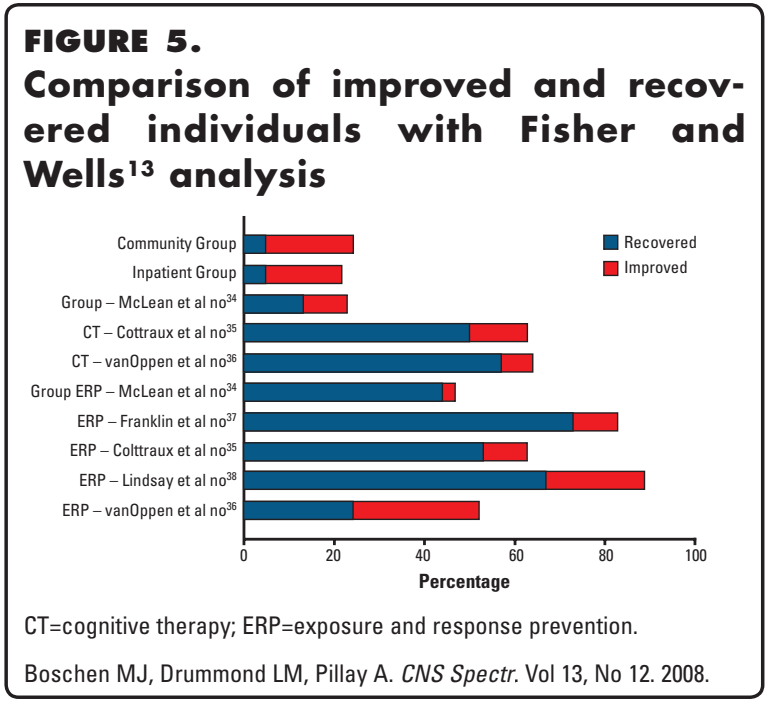

by Eddy and colleauges ${ }^{11}$ in their meta-analysis of OCD treatment studies. The uncontrolled effect size of the community treatment program was 1.22 , which is contrasted with the results of Eddy and colleagues ${ }^{11}$ in Figure 4. The number of community patients who experienced significant improvement in their symptoms, and those who experienced recovery are compared with the results of Fisher and Wells ${ }^{13}$ in Figure 5.

\section{DISCUSSION}

This is the first clinical practice research to examine outcomes, including individual responses to treatment, in a group of individuals with severe, treatment-resistant OCD across inpatient and outpatient settings. We believe our results give considerable cause for hope and optimism for both clinicians and patients dealing with severe, treatment-resistant OCD. Treatment of both the inpatient and community samples showed a statistically significant treatment effect, as well as large effect sizes. Treatment effects were significant regardless of whether they were measured using self-report or clinician-rated instruments. Reductions in OCD symptoms were also accompanied by a reduction in depressive symptoms. As would be expected in a treatmentrefractory group, relatively few patients experienced a fall in symptom severity that would

\section{FIGURE 6. \\ Y-BOCS and BDI Total Scores over treatment for the community sample*}

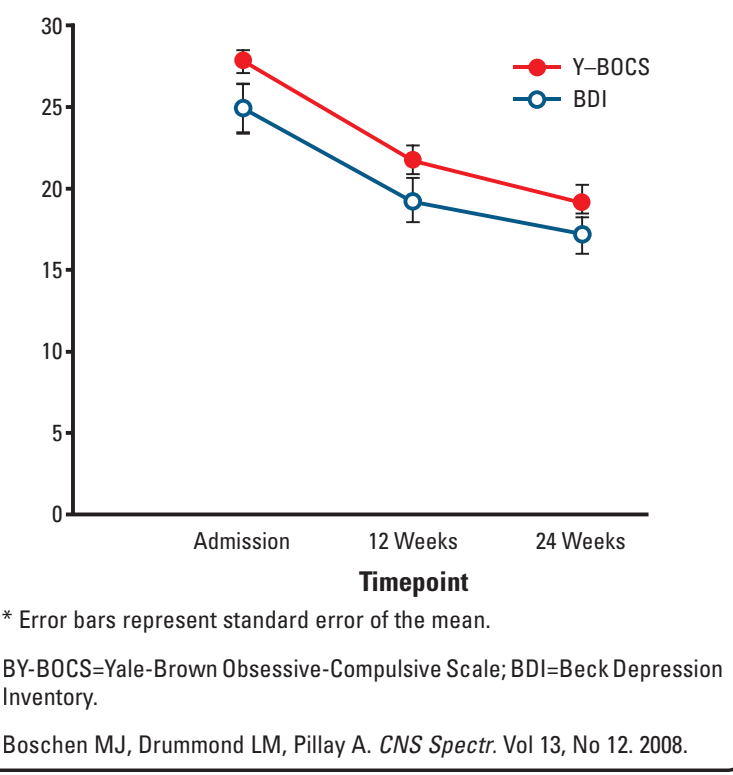


classify them as clinically improved or asymptomatic.

Comparisons between the effect sizes of our inpatient and community treatments and those of the meta-analysis conducted by Eddy and colleagues ${ }^{11}$ provide further support for the use of an intensive treatment regimen. For the inpatient group, the effect size was large, and of a similar magnitude to that reported by previous authors. The reader should, of course, be mindful that effect sizes are often larger when reporting on those with more severe symptoms at the initial assessment. ${ }^{33}$ The effect size for the community cohort also compares favorably with earlier research when one takes into account the nature of the cases treated in our two cohorts. These effect sizes are particularly impressive when one considers that the previously calculated effect sizes from meta-analyses" ${ }^{11}$ are derived from efficacy studies using participants with strict inclusion and exclusion criteria. Our participants had already been trialled using several previous interventions, and failed to experience significant improvement. Because of this, our effect size measures can tentatively be viewed as the size of the treatment effect in addition to those from previous therapy.

As would be expected in a severe, treatment-resistant group, comparatively few individuals experienced a degree of change in OCD symptoms that would classify them as clini-

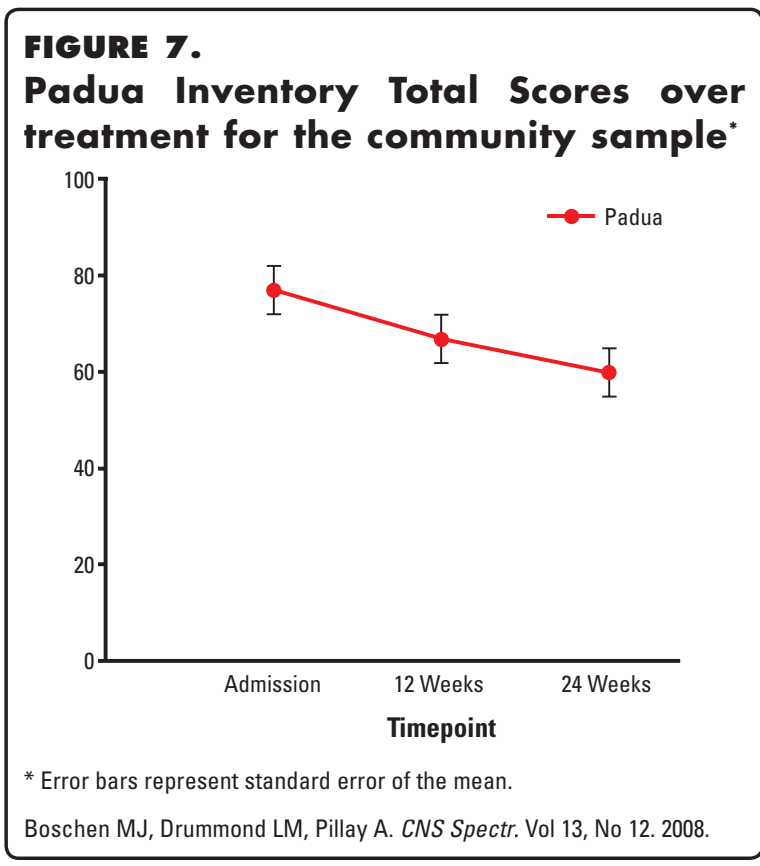

cally improved or asymptomatic. Comparisons between recovery rates in our two samples and those of previous treatment studies demonstrated poorer results for our inpatient and community samples, particularly when looking at the percentage of individuals who were recovered/asymptomatic. Complete recovery from OCD in a previously refractory group of patients is likely to be difficult to attain, even by a specialist, intensive, inpatient treatment service. Despite this, however, the change seen in a notable percentage of those treated gives cause for cautious optimism in this challenging group. For individuals with severe OCD that have not been responsive to several previous treatment attempts, a drop $\geq 10$ points (as occurred in $44 \%$ of our inpatient sample, and $40 \%$ of our community sample) is welcome, even if it does not mean a complete remission of their disorder.

Although the use of different outcome measures makes direct comparisons with the cohort treated by Drummond ${ }^{19}$ or Thornicroft and colleagues ${ }^{20}$ difficult, our results seem to accord with those found in these earlier studies. All studies examined a group of the most challenging treatment cases, and both found that inpatient, intensive behavioral interventions can be successful when previous treatment has failed.

One broad implication of our results is that for individuals who have not responded to previous psychological and psychiatric intervention, there may still be benefit in considering more intensive treatment options, including inpatient admission for several months. All of our patients had previously been unresponsive or noncompliant with previous treatment regimes, yet many experienced improvement when provided with a more intensive treatment focusing on ERP. For a core group of individuals with the most severe and refractory symptoms, outpatient treatment in non-specialist services may be inadequate.

Treatment gains can also be seen in treatment-resistant cases of OCD when treated by specialist services in the community. The cohort treated in the community show statistically significant improvement after 12 weeks of outpatient treatment, as well as a further significant decline in OCD symptoms after an additional 12 weeks of treatment. Where hospitalization is unnecessary, it appears that severe, chronic OCD can be effectively treated in the community.

Our results also provide support for persistence with treatment strategies, such as ERP, 
even when previous treatment in less intensive modes, have not been effective. Few patients had their previous medication regimes changed, with the emphasis being on intensive use of behavioural treatment strategies in both the community and inpatient groups. Our results suggest that rather than changing to less well-supported interventions, clinicians may be advised to increase the intensity of those strategies with demonstrated efficacy, such as ERP.

It is noteworthy that both the current treatment cohorts are drawn from routine clinical practice, rather than carefully controlled, exclusive treatment settings. Our data provides support for the effectiveness as well as the efficacy of behavioral interventions for OCD. We believe it assists in addressing previous critics of efficacy research by demonstrating that research-based efficacy studies do generalize to the clinical practice setting, even for severe, treatment-refractory cohorts.

Despite the optimism that may arise out of the current findings, the reader should be aware of limitations in the current research. Our study was conducted without the typical methodological rigor seen in research settings. We did not employ the use of a control group, and the treatment protocol was allowed to vary in a way that was deemed by the treating clinical team to best suit the idiosyncratic needs of each individual patient. Furthermore, there is not yet data supporting the long-term impact of the inpatient and community interventions, although collection of this information is currently underway. Despite this, we believe our results provide a valuable insight into the effectiveness of intensive treatment of difficult cases of OCD.

The reader should also be mindful of the costs of intensive inpatient or community treatment by specialist treatment teams. Inpatient admission, in particular, is comparatively expensive, and we have not undertaken an analysis of the cost-effectiveness of our treatment. Even so, the intensive treatment methods reported here are reserved for the most severe, chronic, treatment-resistant cases of OCD, where all previous treatments have failed. We suggest that such treatment represents the final step of a steppedcare model described in the NICE guidelines for OCD treatment. ${ }^{32}$ Reserving intensive treatments, such as those described here, to the most severe cases ensures that cost of treatment services is kept to a minimum.

\section{CONCLUSION}

We believe that the results of our community and inpatient intervention programs at the BCPU are a source of optimism for clinicians and patients dealing with severe, treatment-resistant OCD. Even in those who have not responded to earlier interventions with either psychological or psychopharmacologic treatments, there may still be benefit in more intensive, specialist treatment regimes. CNS

\section{REFERENCES}

1. Diagnostic and Statistical Manual of Mental Disorders. 4th ed, text rev. Washington, DC: American Psychiatric Association: 2000.

2. Kessler RC, Berglund P, Demler D, Jin R, Walters EE. Lifetime prevalence and age-of-onset distributions of DSM-IV disorders in the National Comorbidity Survey replication. Arch Gen Psychiatry. 2005;62:593-602.

3. Andrews G, Creamer M, Crino R, Hunt C, Lampe L, Page A. The Treatment of Anxiety Disorders: Clinician Guides and Patient Manuals. 2nd ed. New York, NY: Cambridge University Press; 2002

4. Hendersen S, Andrews G, Hall W. Australia's mental health review: an overview of the general population survey. Aust N Z J Psychiatry. 2000;34:197-205.

5. Riggs DS, Hiss H, Foa EB. Marital distress and the treatment of obsessive-compulsive disorder. Behav Ther. 1992;23:585-597.

6. Leon AC, Portera L, Weissman MM. The social costs of anxiety disorders. Br J Psychiatry Suppl. 1995;27:19-22.

7. Eisen JL, Mancebo MA, Pinto A, et al. Impact of obsessive-compulsive disorder on quality of life. Compr Psychiatry. 2006;47:270-275.

8. Norberg MM, Calamari JE, Cohen RJ, Riemann BC. Quality of life in obsessivecompulsive disorder: an evaluation of impairment and a preliminary analysis of the ameliorating effects of treatment. Uepress Anxiety. 2008;25:248-259.

9. Olatunji BO, Cisler JM, Tolin DF. Quality of life in the anxiety disorders: a metaanalytic review. Clin Psychol Rev. 2007;27:572-581.

10. Boschen MJ. Publication trends in individual anxiety disorders: 1980-2015. J Anxiety Disord. 2008;22:570-575

11. Eddy KT, Dutra L, Bradley R, Westen D. A multidimensional meta-analysis of psychotherapy and pharmacotherapy for obsessive-compulsive disorder. Clin Psychol Rev. 2004;24:1011-1030

12. Jacobsen NS, Truax P. Clinical significance: a statistical approach to defining meaningful change in psychotherapy research. J Consult Clin Psychology. 1991;59:12-19.

13. Fisher PL, Wells A. How effective and cognitive and behavioral treatments for obsessive-compulsive disorder? A clinical significance analysis. Behav Res Ther. 2005; $43: 1543-1558$

14. Borkovec TD, Castonguay LG. What is the scientific meaning of empirically supported therapy? J Consult Clin Psychol. 1998;66:136-142.

15. Goldfried MR, Wolfe BE. Toward a more clinically valid approach to therapy research. J Consult Clin Psychol. 1998;66:143-150.

16. Persons JB. Psychotherapy outcome studies do accurately represent current models of psychotherapy: a proposed remedy. Am Psychol. 1991;46:99-106.

17. Kessler RC, Chiu WT, Demler O, Walters EE. Prevalence, severity, and comorbidity of 12-month DSM-IV disorders in the National Comorbidity Servey replication. Arch Gen Psychiatry. 2005;62:617-627.

18. Jacobsen NS, Roberts LJ, Berns SB, McGlinchey JB. Methods for defining and determining the clinical significance of treatment effects: Description, applications, and alternatives. J Consult Clin Psychol. 1999;67:300-307.

19. Drummond LM. The treatment of severe, chronic, resistant obsessive-compulsive disorder. An evaluation of an in-patient programme using behavioural psychotherapy in combination with other treatments. Br J Psychiatry. 1993;163:223-229.

20. Thornicroft G, Colson L, Marks I. An in-patient behavioural psychotherapy unit: description and audit. Br J Psychiatry. 1991;158:362-367.

21. Stewart SE, Stack DE, Farrell C, Pauls DL, Jenike MA. Effectiveness of intensive residential treatment (IRT) for severe, refractory obsessive-compulsive disorder. $J$ Psychiatr Res. 2005;39:603-609.

22. Goodman WK, Price LH, Rasmussen SA, et al. The Yale-Brown Obsessive Compulsive Scale: II. Validity. Arch Gen Psychiatry. 1989;46:1012-1016.

23. Goodman WK, Price LH, Ramussen SA, et al. The Yale-Brown ObsessiveCompulsive Scale: I. Development, use and reliability. Arch Gen Psychiatry. 1989b;46:1006-1011

24. Pallanti S, Hollander E, Bienstock C, et al. Treatment non-response in OCD: methodological issues and operational definitions. Int $\mathrm{J}$ Neuropsychopharmacol. 2002:5:181-191. 
25. Drummond LM, Pillay A, Rani RS, Kolb P. Specialised Inpatient treatment for severe, chronic, resistant obsessive-compulsive disorder (OCD): a naturalistic study of clinical outcomes. Psychiatr Bull. 2007;31:49-52.

26. Drummond LM, Fineberg NA, Heyman I, et al. Description of progress in the development of a national service for adolescents and adults with the most severe, refractory obsessive-compulsive and body dysmorphic disorders. Psychiatr Bull. 2008:32:333-336.

27. Sanavio S. Obsessions and compulsions: the Padua Inventory. Behav Res Ther. 1988;26:169-177.

28. Beck AT. Beck Depression Inventory Manual. San Antonio, TX: Psychological Corporation; 1993.

29. Beck AT, Ward CH, Mendelson M, Mock J, Erlbaugh J. An inventory for measuring depression. Arch Gen Psychiatry. 1961;4:53-61.

30. Beck AT, Steer RA, Garbin MG. Psychometric properties of the Beck Depression Inventory: twenty-five years of evaluation. Clin Psychol Rev. 1998;8: 77-100.

31. Drummond LM, Pillay A, Kolb P, et al. The introduction of a community model for the treatment of obsessive-compulsive and body dysmorphic disorders. Psychiatr Bull. 2008;32:336-340.

32. British Psychological Society, Royal College of Psychiatrists. Obsessive-compulsive disorder: core interventions in the treatment of obsessive-compulsive disorder and body dysmorphic disorder. London, UK: 2006. National Clinical Practice Guideline Number 31.

33. Westbrook D, Kirk J. The clinical effectiveness of cognitive behaviour therapy: outcome for a large sample of adults treated in routine practice. Behav Res Ther. 2005:43:1243-1261.

34. McLean PD, Whittal ML, Thordarson DS, et al. Cognitive versus behavior therapy in the group treatment of obsessive-compulsive disorder. J Consult Clin Psychol. 2001:69:205-214.

35. Cottraux J, Note I, Yao SN, et al. A randomized controlled trial of cognitive therapy versus intensive behavior therapy in obsessive-compulsive disorder. Psychother Psychosom. 2001;70:288-297.

36. van Oppen P, De Haan E, van Balkom AJLM, Spinhoven P, Hoogduin K, van Dyck R. Cognitive therapy and exposure in vivo in the treatment of obsessive compulsive disorder. Behav Res Ther. 1995;33:379-390.

37. Franklin ME, Abramowitz JS, Kozak MJ, Levitt JT, Foa EB. Effectiveness of exposure and ritual prevention for obsessive-compulsive disorder: randomized compared with nonrandomized samples. J Consult Clin Psychol. 2000;68:594-602.

38. Lindsay M, Crino R, Andrews G. Controlled trial of exposure and response prevention in obsessive-compulsive disorder. Br J Psychiatry. 1997;171:135-139. 